Panagiotis Antsaklis, Konstantinos Tasias*, Alexandros Psarris, Marianna Theodora, George Daskalakis and Alexandros Rodolakis

\title{
Covid-19 and pregnancy: the experience of a tertiary maternity hospital
}

https://doi.org/10.1515/jpm-2021-0070

Received February 6, 2021; accepted April 14, 2021;

published online June 29, 2021

\section{Abstract}

Objectives: The aim of our study is to present the effect of severe acute respiratory syndrome coronavirus 2 (SARS-CoV-2) infection in pregnancy and evaluate its impact on maternal and neonatal outcomes.

Methods: We retrospectively reviewed the medical records of all COVID-19 positive pregnant women who were admitted to "Alexandra" maternity hospital from March to December $2020(n=40)$. The infection status was determined via real-time polymerase chain reaction (RT-PCR) of nasopharyngeal swab specimen. We documented the demographic features, clinical status, pregnancy characteristics and maternal and fetal outcomes.

Results: Forty COVID-19 positive pregnant women were admitted to our clinic during the study period. Mean maternal age was 27.6 years. Gestational age (GA) ranged from 10-42 weeks of pregnancy with mean GA calculated at $38^{+3}$ weeks. Associated comorbidities included hypertension, hypothyroidism, epilepsy, hepatitis B and asthma. Thirty-five out of 40 women delivered within the study period. Cesarean section was performed in $57.1 \%$ of the cases. Most of the cases $(87.5 \%)$ were asymptomatic while ventilation was required for only one patient. All neonates tested negative for SARS-CoV-2. Neither maternal nor neonatal deaths occurred.

Conclusions: In alignment to other studies, our data show that the course of coronavirus disease 2019 (COVID-19)

*Corresponding author: Konstantinos Tasias, MD, 1st Department of Obstetrics and Gynecology, "Alexandra" General Hospital, National and Kapodistrian University of Athens, Vasilissis Sofia 80 and Lourou Street, 11528 Athens, Greece, Phone: +30 6947524456,

E-mail: tasias.konstantinos@gmail.com

Panagiotis Antsaklis, Alexandros Psarris, Marianna Theodora, George Daskalakis and Alexandros Rodolakis, 1st Department of Obstetrics and Gynecology, "Alexandra” Maternity Hospital, National and Kapodistrian University of Athens, Athens, Greece.

https://orcid.org/0000-0001-8280-7796 (M. Theodora) during pregnancy in the majority of cases is mild and neonatal outcomes also appear favorable.

Keywords: COVID-19; maternal outcome; neonatal outcomes; obstetric outcomes; pregnancy; SARS-CoV-2.

\section{Introduction}

In December 2019, a pneumonia caused by novel coronavirus (severe acute respiratory syndrome coronavirus 2 [SARS-CoV-2]) emerged in Wuhan, the capital of Hubei province, in China [1]. Since then, the infection has spread to different areas of China and other parts of the world [1]. World Health Organization (WHO) announced SARS-CoV-2 as cause of pneumonia on January 9, 2020, and declared a pandemic on March 11, 2020 [2]. The SARS-CoV-2 causes the coronavirus disease 2019 (COVID-19) with symptoms ranging from mild disease to pneumonitis, acute respiratory distress syndrome (ARDS) and death. Globally, as of 6 February 2021, 104,790,123 cases of COVID-19 have been reported, including 2,285,048 deaths [3]. The first reported case in Greece was reported on the 27th of February 2020. Since then, there have been 160,935 confirmed cases and 5,903 deaths from COVID-19 [3].

Inevitably the number of COVID-19 positive pregnant women is rising on par with the general population. At the same time, information about the effects of COVID-19 during pregnancy is evolving rapidly. Initial reports suggested that the severity of the illness in pregnant patients is similar with the general population [4, 5]. However, later reports suggested higher rates of intensive care unit admissions, invasive ventilation and mortality among pregnant patients compared to the general population [6]. Pneumonia remains one of the leading causes of pregnant women mortality worldwide [7]. Pregnant women are considered high risk for viral infections, such as SARS-CoV-2, due to the effects of pregnancy on the immunity response [8]. Furthermore, there are concerns regarding the association of SARS-CoV-2 and fetal growth restriction (FGR) after maternal infection, due to placental lesions related to acute illness, increased coagulopathy, hypoxia and placental 
viral infection which have been observed in numerous occasions [9-13]. Hence, management of COVID-19 positive pregnant women remains challenging.

The objective of our study is to present the maternal and neonatal outcomes of COVID-19 positive pregnant women, who were admitted to our tertiary maternity hospital since the beginning of the pandemic in Greece.

\section{Materials and methods}

All COVID-19 positive pregnant women who were admitted to our clinic from March to December 2020 were included in the study. We documented the demographic data, clinical characteristics, laboratory values, maternal and fetal outcomes. More specifically we documented maternal age, body mass index (BMI), blood group, gravidity, parity, indication for admission, comorbidities, gestational age (GA), pregnancy complications, initial COVID-19 symptoms, hematological and biochemical results, indication and mode of delivery, hospitalization length and final outcome. Similarly, we documented neonatal birthweight, sex, Apgar score, neonatal COVID-19 status, neonatal morbidity, neonatal intensive care (NICU) admission and final outcome.

Assessment of SARS-CoV-2 in nasopharyngeal samples was performed by real-time polymerase chain reaction (RT-PCR) targeting the ribonucleic acid (RNA)-dependent RNA polymerase ( $R d R p)$ gene. Sterile Dacron or Rayon swabs with flexible plastic shafts were used to collect nasopharyngeal samples from patients. After collection, swabs were placed into $2 \mathrm{~mL}$ of sterile viral transport medium (VTM; various manufacturers) and all specimens were analyzed by the university laboratory.

Informed consent was obtained from all pregnant women included in this study. Cumulative data of continuous variables was reported in mean $\pm \mathrm{SD}$.

Ethical approval: Research relating to human subjects complied with national regulations, institutional policies the tenets of the Helsinki Declaration [14], and has been approved by the authors' hospital Institutional Review Board.

\section{Results}

A total of 40 pregnant women tested positive for COVID-19 from March till December 2020 and were included in our study. Mean age at the time of diagnosis was 28 years (SD 6.3 years) and mean BMI was $26.2 \mathrm{~kg} / \mathrm{m}^{2}$. Mean GAe at diagnosis was $37^{+3}$ weeks and 38 of them were at the third trimester. The majority of women $(n=35 / 40)$ were asymptomatic at the time of diagnosis and remained asymptomatic throughout the study period. About $27 \%$ of the study population $(\mathrm{n}=12 / 40)$ had comorbidities, such as morbid obesity (BMI $51.3 \mathrm{~kg} / \mathrm{m}^{2}$ ), gestational diabetes mellitus (GDM), asthma, epilepsy, hepatitis B, hypothyroidism and thrombophilia. Initial symptoms included cough, sore throat, fever and dyspnea. A small percentage of symptomatic pregnant women (5\%) needed oxygen, while none of the women were admitted to the Intensive Care Unit (ICU). Chest radiograph was performed in two cases and one had radiographic findings suspicious for COVID-19 pneumonia. The most common blood group among positive pregnant women was $\mathrm{A}+$ and the mean leukocyte count was $10.1 \times 10^{6} / \mathrm{mm}^{3}$ (SD 3.3). Demographic features and clinical characteristics are shown in Table 1.

\section{Pregnancy outcomes}

Obstetric and neonatal outcomes are shown in Table 2. Thirty-five women delivered during the study period. Cesarean section was performed in $57.1 \%$ of the cases, compared to the annual rate of cesarean section for pregnant women without COVID-19 in out hospital which is $48 \%$, while 15 women had vaginal deliveries. GA at delivery ranged from 34 to $41^{+4}$ weeks with a mean $\mathrm{GA} 38^{+3}$ weeks. Pregnancy related complications occurred in 16 women. The most common pregnancy related complication in our study population was GDM with a frequency of $17.5 \%$ $(n=7)$. Six women delivered before 37 weeks, half of them due to intrauterine growth restriction (IUGR) causing fetal distress $(n=3)$ and the other half due to premature rapture of membranes (PROM) $(n=3)$. As for the neonates, 35 neonates were delivered during the study period. All neonates tested negative for SARS-CoV-2 via RT-PCR. Birth weight ranged from 1,580 to $4,420 \mathrm{~g}$ with a mean weight of 3,153.7 g. Apgar score ranged from 6 to 9 at the 1st min while the median Apgar score at the 5th min was 10. One neonate was admitted to the Neonatal Intensive Care Unit (NICU) due to meconium aspiration syndrome and pulmonary hypertension. Twenty-two out of 35 neonates breastfed and none were separated from their mothers. Neither maternal nor neonatal deaths occurred in our study population.

\section{Discussion}

Our study has demonstrated that COVID-19 during pregnancy in the majority of cases has mild clinical course. A high rate of asymptomatic COVID-19 positive pregnant women was documented $(n=35 / 40)$, as expected by the relatively high documented number of asymptomatic pregnancies [15]. As COVID-19 positive asymptomatic individuals pose a great risk for spreading the virus to other patients and healthcare providers, universal testing of 
Table 1: Demographic features and clinical characteristics of for SARS-CoV-2 positive cases $(n=40)$.

\begin{tabular}{|c|c|}
\hline Variables & Values \\
\hline Maternal age, years & $\begin{array}{l}28 \pm 6.3(18-45), \text { median } \\
28.5\end{array}$ \\
\hline Gravidity & $2(1-5)$, median 2 \\
\hline Parity & $1(0-6)$, median 1 \\
\hline Previous miscarriage & $0(0-2)$, median 0 \\
\hline $\mathrm{BMI}, \mathrm{kg} / \mathrm{m}^{2}$ & $\begin{array}{l}26.2(18-51.8), \text { median } \\
25.1\end{array}$ \\
\hline Comorbid disease & $12(30 \%)$ \\
\hline Smoking & $3(7.5 \%)$ \\
\hline Hypothyroidism & $2(5 \%)$ \\
\hline Asthma & $1(2.5 \%)$ \\
\hline Hepatitis B & $1(2.5 \%)$ \\
\hline Epilepsy & $1(2.5 \%)$ \\
\hline Thrombophilia & $1(2.5 \%)$ \\
\hline Gestational age at diagnosis, weeks & $\begin{array}{l}37^{+} 3\left(10-41^{+4}\right), \text { median } \\
38^{+} 5\end{array}$ \\
\hline \multicolumn{2}{|l|}{ Pregnancy trimester at diagnosis } \\
\hline First & $1(2.5 \%)$ \\
\hline Second & $1(2.5 \%)$ \\
\hline Third & $38(95 \%)$ \\
\hline Asymptomatic & $35(87.5 \%)$ \\
\hline Initial symptoms & $5(12.5 \%)$ \\
\hline Fever & $1(2.5 \%)$ \\
\hline Cough & $2(5 \%)$ \\
\hline Sore throat & $2(5 \%)$ \\
\hline Dyspnea & $1(2.5 \%)$ \\
\hline \multicolumn{2}{|l|}{ Medications } \\
\hline Low molecular weight heparin & $2(5 \%)$ \\
\hline Insulin & $4(10 \%)$ \\
\hline Aspirin & $2(5 \%)$ \\
\hline Progesterone & $1(2.5 \%)$ \\
\hline Additional pathogen & $1(2.5 \%)$ \\
\hline \multicolumn{2}{|l|}{ Respiratory support } \\
\hline Nasal oxygen therapy & $1(2.5 \%)$ \\
\hline Non-invasive ventilation & $1(2.5 \%)$ \\
\hline ICU admission & $0(0 \%)$ \\
\hline Length of hospital stay, days & $5(1-16)$ \\
\hline \multicolumn{2}{|l|}{ Blood group } \\
\hline$A+$ & $15(37.5 \%)$ \\
\hline$A-$ & $1(2.5 \%)$ \\
\hline $\mathrm{B}+$ & $9(22.5 \%)$ \\
\hline B- & $1(2.5 \%)$ \\
\hline$A B+$ & $3(7.5 \%)$ \\
\hline$A B-$ & $0(0 \%)$ \\
\hline $0+$ & $10(25 \%)$ \\
\hline $0-$ & $1(2.5 \%)$ \\
\hline Leukocyte, $10^{6} / \mathrm{mm}^{3}$ & $\begin{array}{l}10.1 \pm 3.3(4.4-19.5) \\
\text { median } 9.7\end{array}$ \\
\hline Neutrophil percentage, \% & 75 (53.6-88.7), median 77 \\
\hline Radiologic imaging & $2(5 \%)$ \\
\hline $\begin{array}{l}\text { Radiologic imaging findings suspicious } \\
\text { for COVID-19 }\end{array}$ & $1(2.5 \%)$ \\
\hline
\end{tabular}

SARS-CoV-2, severe acute respiratory syndrome coronavirus 2; ICU, intensive care unit; COVID-19, coronavirus disease 2019.
Table 2: Obstetric and neonatal outcomes of SARS-CoV-2 positive cases $(n=40)$.

\begin{tabular}{ll}
\hline Variables & Cases (n=40) \\
\hline Pregnancy complications & $16(40 \%)$ \\
FGR & $3(7.5 \%)$ \\
GDM & $7(17.5 \%)$ \\
Oligohydramnios & $3(7.5 \%)$ \\
Hydramnios & $1(2.5 \%)$ \\
Macrosomia & $1(2.5 \%)$ \\
PROM & $2(5 \%)$ \\
Preterm delivery & $6(15 \%)$ \\
Pre-eclampsia & $1(2.5 \%)$ \\
Gestational hypertension & $2(5 \%)$ \\
Leukocytosis & $1(2.5 \%)$ \\
Preterm contractions & $3(7.5 \%)$ \\
Cervical inadequacy & $1(2.5 \%)$ \\
Placenta accreta/previa & $1(2.5 \%)$ \\
Delivery status & \\
Ongoing pregnancy & $5(12.5 \%)$ \\
Delivered & $35(87.5 \%)$ \\
Route of delivery & $15(42.9 \%)$ \\
Vaginal delivery & $20(57.1 \%)$ \\
Cesarean section & $10(6-10)$, median 10 \\
Cesarean indications & $2(5.7 \%)$ \\
Previous cesarean section & $5(0 \%)$ \\
Fetal distress & $22(62.8 \%)$ \\
Macrosomia & $5(25 \%)$ \\
Failure to progress & $1(5 \%)$ \\
Breech presentation & $4(20 \%)$ \\
Pre-eclampsia & $2(10 \%)$ \\
Epilepsy & $1(5 \%)$ \\
Pregnant choice/IVF & $1(5 \%)$ \\
Gestational age at delivery, weeks & $38^{+3}\left(34-41^{+4}\right)$, median $39^{+} 1$ \\
Birth weight, g & $3,153.7 \pm 590.74(1,580-4,420)$, \\
Apgar score 1st min & median 3,105 \\
Apgar score 5th min & $8(6-9)$, median 9 \\
Admission to NICU & \\
Neonatal SARS-CoV-2 positivity & \\
\hline Freastfeeding & \\
\hline
\end{tabular}

FGR, fetal growth restriction; GDM, gestational diabetes mellitus; PROM, premature rapture of membranes; NICU, neonatal intensive care unit.

hospitalized pregnant women on the day of admission has been adopted as a standard protocol.

Women in labor or needing immediate medical attention were divided in two categories while the COVID-19 test results were pending. Asymptomatic women, without any contact with COVID-19 patients in the near past were characterized as low-risk and treated as usual. Women who presented with symptoms such as fever, cough, dyspnea, had close contact with confirmed COVID-19 positive patients or resided in closed communities (such as prisons, institutions and immigration camps) with recent COVID-19 
cases, were characterized as high risk for COVID-19 infection and they were examined or delivered in a separate hospital ward.

High risk pregnant women whose RT-PCR COVID-19 test result was pending were kept in isolation.

Hospital infrastructure was modified in order to adopt the necessary changes to accommodate, treat and deliver COVID-19 positive pregnant women. Aeration of the operating room was stopped to limit the spread within the hospital. One ward was allocated for the hospitalization of COVID-19 positive women and another ward for the high risk pregnant women whose RT-PCR test was pending. A separate ward was used for delivering or operating on the high risk or positive pregnant patients.

Hospital policy dictated that the patient wore a surgical mask throughout the duration of hospital stay. Hand hygiene, use of N-95/FFP2/FFP3 masks, face shields, feet protection, two sets of gloves and Tyvek or waterproof uniforms were used for protection of the hospital staff when taking care of COVID-19 positive or high risk women.

Breastfeeding was encouraged in all cases, while COVID-19 positive mothers were instructed regarding proper hygiene.

None of the 35 neonates who were delivered from COVID-19 positive women tested positive for SARS-CoV-2. However, perinatal complications were reported in some cases. We documented high rates of FGR $(n=3 / 40)$ but the presence of concurrent maternal pathology (gestational hypertension, GDM etc.) does not allow for speculation regarding the etiology of FGR.

Breastfeeding was encouraged in our department as there are no evidence regarding transmission of SARS-CoV2 through the breast milk $[16,17]$. As a result 22 out of 35 women elected to breastfeed with none of the infants testing positive of COVID-19 until discharge from the hospital, approximately three to 4 days post-delivery.

As this global epidemic continues to develop, additional information regarding the effects of COVID-19 on pregnant women and their infants will become available. Recommendation on the subject of obstetric management during the pandemic are available from most Obstetric Colleges and Associations and they are being updated regularly as new information becomes available [18-20].

Vaccination against COVID-19 has begun in the United States of America since the 11th of December 2020, when the Food and Drug Administration issued an Emergency Use Authorization for the (BNT162b2) messenger ribonucleic acid (mRNA) vaccine in individuals 16 years of age and older [21]. Despite the promising results of vaccination (which has been shown to be $95 \%$ effective at preventing COVID-19), pregnant women were not included in the vaccine's clinical trials [21]. However, on the 13th of December 2020 the American College of Obstetricians and Gynecologists issued a practice advisory supporting vaccination of pregnant women against COVID-19 after informing the pregnant women of the safety of mRNA vaccines in pregnancy and the risks of maternal disease to the mother and the fetus [22]. According to Society of Obstetricians and Gynecologists of Canada, pregnant and breastfeeding women were excluded from the Phase II and Phase III studies for the COVID-19 vaccine. However, there were 23 women ( 12 in the vaccine arm and 11 in the placebo arm) who reported pregnancies during the trial and are being followed for pregnancy outcomes with no reports of adverse effects [23].

\section{Conclusions}

In accordance with other studies, COVID-19 infection causes mild-to-moderate symptoms while many infected individuals are asymptomatic. As our knowledge regarding COVID-19 infection of pregnant women is being updated on a daily basis, there is need for flexibility regarding the allocation of resources, equipment and healthcare workforce. Data regarding first and second trimester infection is insufficient for optimal management. More data and follow up are still needed.

Research funding: None declared.

Author contributions: All authors have accepted responsibility for the entire content of this manuscript and approved its submission.

Competing interests: Authors state no conflict of interest. Informed consent: Informed consent was obtained from all individuals included in this study.

Ethical approval: Research relating to human subjects complied with national regulations, institutional policies the tenets of the Helsinki Declaration [14], and has been approved by the authors' hospital Institutional Review Board.

\section{References}

1. Zhu N, Zhang D, Wang W, Li X, Yang B, Song J, et al. A novel coronavirus from patients with pneumonia in China, 2019. N Engl J Med 2020;382:727-33.

2. WHO. Director-General's opening remarks at the media briefing on COVID-19. Available from: https://www.who.int/director-general/ speeches/detail/who-director-general-s-opening-remarks-at-themedia-briefing-on-covid-19-11-march-2020 [Accessed 24 Dec 2020]. 
3. WHO. Coronavirus disease (COVID-19) dashboard. Available from: https://covid19.who.int/ [Accessed 24 Dec 2020].

4. Novel coronavirus 2019 (COVID-19). ACOG. Available from: https://www.acog.org/clinical/clinical-guidance/practiceadvisory/articles/2020/03/novel-coronavirus-2019 [Accessed 24 Dec 2020].

5. CDC COVID-19 Response Team. Preliminary estimates of the prevalence of selected underlying health conditions among patients with coronavirus disease 2019 - United States, February 12-March 28, 2020. MMWR Morb Mortal Wkly Rep 2020;69: 382-6.

6. Zambrano LD, Ellington S, Strid P, Galang RR, Oduyebo T, Tong VT, et al. Update: characteristics of symptomatic women of reproductive age with laboratory-confirmed SARS-CoV-2 infection by pregnancy status - United States, January 22October 3, 2020. MMWR (Morb Mortal Wkly Rep) 2020;69: 1641-7.

7. Dashraath P, Wong JIJ, Lim MXK, Lim LM, Li S, Biswas A, et al. Coronavirus disease 2019 (COVID-19) pandemic and pregnancy. Am J Obstet Gynecol 2020;222:521-31.

8. Warning JC, McCracken SA, Morris JM. A balancing act: mechanisms by which the fetus avoids rejection by the maternal immune system. Reproduction 2011;141:715-24.

9. Kirtsman M, Diambomba Y, Poutanen SM, Malinowski AK, Vlachodimitropoulou E, Parks WT, et al. Probable congenital SARS-CoV-2 infection in a neonate born to a woman with active SARS-CoV-2 infection. CMAJ (Can Med Assoc J) 2020;192:E647-50.

10. Vivanti AJ, Vauloup-Fellous C, Prevot S, Zupan V, Suffee C, Do Cao J, et al. Transplacental transmission of SARS-CoV-2 infection. Nat Commun 2020;11:1-3572.

11. Prabhu M, Cagino K, Matthews KC, Friedlander RL, Glynn SM, Kubiak JM, et al. Pregnancy and postpartum outcomes in a universally tested population for SARS-CoV-2 in New York City: a prospective cohort study. BJOG An Int J Obstet Gynaecol 2020; 127:1548-56.

12. Shanes ED, Mithal LB, Otero S, Azad HA, Miller ES, Goldstein JA, et al. Placental pathology in COVID-19. Am J Clin Pathol 2020;154: 23-32.

13. Smithgall MC, Liu-Jarin X, Hamele-Bena D, Cimic A, Mourad M, Debelenko $\mathrm{L}$, et al. Third-trimester placentas of severe acute respiratory syndrome coronavirus 2 (SARS-CoV-2)-positive women: histomorphology, including viral immunohistochemistry and in-situ hybridization. Histopathology 2020;77:994-9.

14. World Medical Association. World Medical Association Declaration of Helsinki: ethical principles for medical research involving human subjects. J Am Med Assoc 2013;310:2191-4.

15. Sutton D, Fuchs K, D'Alton M, Goffman D. Universal screening for SARS-CoV-2 in women admitted for delivery. N Engl J Med 2020; 382:2163-4.

16. Chen H, Guo J, Wang C, Luo F, Yu X, Zhang W, et al. Clinical characteristics and intrauterine vertical transmission potential of COVID-19 infection in nine pregnant women: a retrospective review of medical records. Lancet 2020;395:809-15.

17. RCOG. Coronavirus (COVID-19) infection and pregnancy. Available from: https://www.rcog.org.uk/coronaviruspregnancy [Accessed 24 Dec 2020].

18. RCOG. Coronavirus (COVID-19) infection in pregnancy. Information for healthcare professionals, Version 13; 2021. https://www.rcog.org.uk/globalassets/documents/guidelines/ 2021-02-19-coronavirus-covid-19-infection-in-pregnancy-v13.pdf [Accessed 19 Feb 2021].

19. Novel Coronavirus 2019 (COVID-19). ACOG. Available from: https:// www.acog.org/clinical/clinical-guidance/practice-advisory/ articles/2020/03/novel-coronavirus-2019 [Accessed 10 Jan 2021].

20. Api O, Sen C, Debska M, Saccone G, D’Antonio F, Volpe N, et al. Clinical management of coronavirus disease 2019 (COVID-19) in pregnancy: recommendations of WAPM-World Association of Perinatal Medicine. J Perinat Med 2020;48:857-66.

21. FDA. FDA takes key action in fight against COVID-19 by issuing emergency use authorization for first COVID-19 vaccine. US FDA. Available from: https://www.fda.gov/news-events/pressannouncements/fda-takes-key-action-fight-against-covid-19issuing-emergency-use-authorization-first-covid-19 [Accessed 24 Dec 2020].

22. ACOG. Vaccinating pregnant and lactating patients against COVID 19. Practice advisory. Available from: https://www.acog. org/clinical/clinical-guidance/practice-advisory/articles/2020/ 12 /vaccinating-pregnant-and-lactating-patients-against-covid19 [Accessed 24 Dec 2020].

23. Schalkwyk V. SOGC statement on COVID-19 vaccination in pregnancy; 2020. Available from: https://sogc.org/common/ Uploaded\%20files/Latest\%20News/SOGC_Statement_COVID19_Vaccination_in_Pregnancy.pdf [Accessed 18 Dec 2020]. 